\title{
THE IMPACT OF ELECTROMAGNETIC FIELDS \& MOBILE TELEPHONES ON HEALTH: IN THE CONTEXT OF SOUTH ASIAN REGIONS
}

Achyuta Nand Mishra and Prof. Dr. Sudan Jha

Dr. K N Modi Univerity, Newai, Rajasthan, India

School of Computer Engineering Kalinga Institute of Industrial Technology, KIIT University Bhubaneswar 751024, Odisha, India

\begin{abstract}
The $20^{\text {th }}$ century witnessed an explosion of technological applications that rely on electricity and thus produce electromagnetic fields. And there will be even more of these innovative technologies in the 21 st century. We cannot avoid this, nor would we wish to, because technology makes our lives healthier, wealthier and safer - it is a major contributor to the economic and social progress that enhances our quality of life. However, while electric fields have been a vital part of our daily life for over a century now, and have not shown clear evidence of ill $\mathrm{e} \square$ ects on health, they are becoming more pervasive. This is seen in the massive growth in the use of mobile telephones - there are 1.6 billion in use today in the world - each one with its own electromagnetic field. This is a subject of growing concern to Europe's citizens who are unsure if mobile telephones are safe for themselves and their children. While there is no proven evidence that very weak electric and magnetic fields can a $\square$ ect our health, we have a duty to be sure of this, so that the public and regulators can make informed decisions about their use. This paper yields out the research into the potential health e $\square$ ects of long-term exposure to electromagnetic fields. Because this research is looking for weak e $\square$ ects that could accumulate over a long time period, actions at the Human level are particularly e $\square$ ective: coordinating new research based on shared knowledge and building co-operation between widespread centers of expertise.
\end{abstract}

Keywords: EMF, Radiation, Digital Effects, Developing World, Socio-Economic problems.

\section{INTRODUCTION}

What are the sources of EMF?

Electromagnetic fields are all around us and in everyday life we are all exposed to EMFs from a variety of sources. There are natural sources such as the Earth's magnetic field and sunlight that contains visible, infrared and ultraviolet frequencies. There are also many man-made sources of non-ionizing EMF generated wherever an electrical current flows. In our homes, EMFs arise from microwave ovens, hairdryers, the electric wiring in the house and remote control devices, among others. In the workplace, We are generated by computer screens, industrial electric furnaces, electric motors, and anti-theft systems. And on the street, we are bathed in weak EMF from electric train and tram cables, power lines, radio-frequency communication antenna for the emergency services, and now, with the growth of wireless networks, by EMFs from Wi-Fi and 'Bluetooth' type technologies. An important feature is that although there are many sources, We are very weak. Mobile telephones are a particular source of EMF that has grown rapidly as people appreciate the many benefits We bring to our lives. Mobile telephone technology generates EMF in two ways: first from the antennae that are placed around our cities, towns and motorways; and secondly from the telephones themselves which transmit our conversations to the antennae. 
How these EMFs interact with biological organisms depends on our energy and frequency. Human bodies are transparent to some frequencies and not to others. For example, sunlight only penetrates 'skin deep' and is mostly absorbed, whereas magnetic fields can largely pass through the human body. Energy is also important, which is why we put on sunscreen creams to protect ourselves in strong sunlight. For similar reasons there are recommended exposure limits for other EMF frequencies, for example the fields emitted by computer screens in workplaces. The following chapter below shows some common domestic sources of EMF and the electric and magnetic fields We generate.

\section{RESEARCH OBJECTIVES - EMF: THE BENEFITS AND CONCERNS}

Some of the non-ionizing fields we encounter in daily life result from the deliberate use of EMF to achieve particular benefits. We are used extensively in medicine for diagnosis and treatment, for example in magnetic resonance scanners to study the brain and in irradiation for bone repair and cancer treatment. A major indirect source of non-ionizing EMFs is the electricity supply grid that we depend on to light buildings and streets, to power our kitchens and televisions, and to run the lifts, trains, computers and industrial machinery that our society needs. Power stations send electricity through overhead and underground cables and substations to our cities, factories and homes - a vital network of flowing electric current that generates EMFs all around us. The fastest growing source of exposure to EMFs is communications, in particular mobile telephony. Although television and radio antennae have been with us for a long time, more recently, the massive growth in mobile telephony is a major success story in which the European GSM standard leads the world. The mobile telephony sector has increased employment in the South Asian Regions; it has improved personal security, in particular for the young; emergency services are faster; business is more $\mathrm{e} \square$ cient; and it helps fulfill our individual need to communicate when and where we want to, by speech, text message, email, and more recently with images.

\section{METHOdOLOGY - A CAUSE FOR CONCERN?}

Exposure to non-ionizing electromagnetic fields is unavoidable in today's society and this exposure is growing mainly because of mobile telephones which are held close to the head, and also to the high density of mobile telephone antennae in our towns and cities. Therefore, the question as to whether or not We can damage our health is an important one. The e $\square$ ects of long-term exposure to low-intensity EMF are not at all well-known - and it is exposure to this type of fields that is growing. The di $\square$ culties in evaluating the e $\square$ ects of long-term low-level exposure to potential environmental hazards are not new; for example, the low concentrations of chemical and biological agricultural residues that get into the food chain have been active research areas for many years. A major problem in this research is that the e $\square$ ects can be cumulative; We build up in the body over time. This means that research into the hazards We pose is long-term and painstaking - and it is further complicated when the long-term research is overtaken by new technologies. Much of this research is into the 'genotoxicity' of ELF and RF-EMF from electricity power lines and mobile telephones respectively. A genotoxin is an agent that can damage DNA and possibly lead to cancer. So far, no convincing links between exposure to low-level EMFs and damage to health have been found.

Awareness of the possible risks of mobile telephones has raised public concern, which has been echoed by the South Asian Countries Parliament. In addition, the spread of antennae through our towns is raising objections, not just for aesthetic reasons but also because of fears about our potential harmful e $\square$ ects. While industry has done much to limit the exposure to EMF from telephones and antennae, public fears are delaying the deployment of next-generation mobile telephone systems.

\section{Nepal's Response to Concerns about EMF: -}

Electricity and EMFs bring countless benefits to society. We cannot do without them, yet we do not know the consequences of long-term exposure to EMFs, if indeed there are any. Therefore, research is needed to understand the risks and set appropriate safety standards. Such research forms part of Research and Technology Development. Eight major projects were funded globally under the Fifth Framework Program (FP5) between 1998 and 2002, and this funding is continuing. The majority of these research projects focus on EMF from mobile telephones and are cancer-related, a smaller number investigate possible effects on hearing, memory and behavior. Research in this field is mainly of three types: in vitro 
research is based on laboratory experiments using biological materials, for example, cell cultures; in vivo research is performed on living organisms, such as rats or humans. These experimental approaches are complemented by epidemiological research that uses surveys and statistics to investigate the occurrence of disease and its relation to environmental factors, for example whether a particular cancer is more common in people living close to communications antennae. A further factor that adds to the time-consuming feature of these research projects is that research results must be replicated by others to confirm the reliability of the conclusions; a single result is not enough. In addition, the research must be published and reviewed so that other researchers can verify or criticize the results.

\section{LITERATURE SURVEY}

Some examples of the projects yielded out in the above mentioned concepts are:

The Reflex project studied how low-energy EMF interacts directly with biological materials in the laboratory (in vitro). The researchers showed that exposing cells to ELF and RF electromagnetic fields could cause DNA to break apart and thus $\mathrm{a} \square$ ect how cells develop. The key to this project lies in the standardized equipment that the partners, from all over Europe, used to do our experiments. This meant that several partners could independently confirm the experimental results. While these results do not prove that there are hazards from EMFs, We do indicate promising lines of investigation for further work.

The Cemfec project looked at how EMFs might interact with known cancer-causing chemicals that can be found in drinking water in minute amounts. The aim was to assess if the EMF frequencies typical of mobile telephones might have an indirect $\mathrm{e} \square$ ect by increasing the genotoxicity of these environmental carcinogens. In this case, the study found that RF-EMF did not enhance the development of cancer.

The Ramp2001 project studies the e $\square$ ect of RF-EMF from mobile telephone handsets on the nervous system, searching for any changes in memory and behavior. By correlating experiments with theoretical modeling, the project tries to identify the mechanisms through which the nervous system could interact with EMF.

The Guard project investigated the e $\square$ ect on hearing of long-term exposure to RF-EMF, using both animal and human studies. Interphone, a project led by the International Agency for Research on Cancer, is collecting extensive epidemiological data on mobile telephone use and the occurrence of cancers in the head and neck, for example brain tumors. Nine EU Member States are participating and parallel studies are running in Australia, Canada, Japan and New Zealand. Concentrating on age groups and regions with a long accumulated usage of mobile telephones, the researchers are using carefully controlled computer-assisted interviews to collect data.

The Perform - A project is using in vivo experiments to investigate whether EMF at mobile telephone frequencies, whether from handsets or antennae, can directly cause cancer in animals or promote the spread of The Fraunhofer Institute for Applied Science in Germany is coordinating the six partners who are also performing important work to verify the results of Australian researchers who found increased cancer incidence in mice exposed to EMF.

A further group of projects focused on evaluating the potential hazards of EMFs used in the workplace, such as in medical diagnosis and from the pulsed electromagnetic fields produced by security systems employed in access control systems and supermarket check outs. Member States also fund our own national research programs on EMFs and health. Many of these programs are coordinated through the framework that coordinates the national research programs on the health implications of mobile telephony of the other countries. At present, 25 countries are cooperating within the framework. This research e $\square$ ort is complemented by a large coordination action, EMF-NET, which brings together European and national EMF projects and researchers as a source of expertise and analysis for policy-makers and the general public.

The aim of EMF-NET is not to produce new research, but the collection and interpretation of existing data from across the world. Given the di $\square$ cult and long-term nature of this research, it is vital that the best use is made of existing information 
and expertise for the benefit of all. EMF-NET also supplies a 'fast-response team' to provide rapid advice on new research results and our relevance to public health and safety issues. EMF-NET also contributes to information activities for the general public. Research into potential health hazards from EMFs has been proposed to continue.

\section{Proposed Research Methodologies}

The issues arising from exposure to environmental electromagnetic fields are of intense public interest, in particular that of whether or not EMF from mobile telephones can damage health. The research needed to clarify these issues is under way and more will be needed in the future. Research into the health e $\square$ ects of long-term, low-level exposure to environmental agents is di $\square$ cult. The work itself can take a significant amount of time, which makes it costly. The e $\square$ ects being investigated are often di $\square$ cult to detect in our early stages, and thus the results must be shown to be reproducible, preferably by other laboratories, again adding to the time and cost. It is for this reason that a coordinated and co-operative research $\mathrm{e} \square$ ort is needed to avoid unnecessary duplication and to ensure that each research activity builds on earlier work. This is why research into the health $\mathrm{e} \square$ ects of EMF benefits particularly from transnational co-operation. Pooling energy and resources, in our regions, funding scientific research into the health e $\square$ ects of EMF exposure involves a broad spectrum of stakeholders. The government plays a key role as the long-term, costly nature of this research makes it vital to encourage cooperation and coordination between developing counties' research teams to avoid repetition, share knowledge and match research competences. The ability to show the reproducibility of di $\square$ cult experiments between laboratories, and the access are real benefits that EU-funded co-operative research provides. The Member State governments play an important role in funding national initiatives and ensuring these e $\square$ orts are complementary to other national research programs. At international level, the EMF program of the WHO ensures that research results are shared worldwide and used to guide a strategic research agenda that sets priorities and avoids unnecessary duplication. The mobile communications industry is also contributing to research funding, driven by the need to ensure safety and gain public acceptance for new technologies and infrastructures. European funding for research on EMF-induced health e $\square$ ects has been proposed by the Commission to continue in the Seventh Framework Programme for RTD that will run from 2007 to 2013. This research will be guided by the knowledge gained in the earlier Framework Program, as well as by results from national and international research e $\square$ orts. The general public's exposure to environmental EMFs is currently defined by the EMF exposure limits advised by the International Commission on Non-Ionizing Radiation Protection.

The Specific Absorption Rate (SAR) measures the amount of energy in watts that a body absorbs per kilogram in an electromagnetic field. For the general public these SAR safety limits have a 50 times safety factor for exposure to EMF from mobile telephones. GSM manufacturers provide public information on the SAR for each of the GSM models We produce. This paper emphasizes on the commitment to reviewing these exposure limits regularly in the light of new research results taking into account the opinions of its Scientific Committees.

\section{Proposed Solution}

The Advice Pulsed Fields project: Do electromagnetic fields generated by security devices have health effects? While public concern about exposure to electromagnetic fields has centered on power lines and mobile telephones, other sources have not attracted the same attention. The Advice Pulsed Fields project looked at a family of sources that have been largely overlooked - security systems that operate by generating pulsed electromagnetic fields. Go to the airport to catch a flight and you know you will be scanned at a security gate before you board. Leave a department store or a library, and the chances are you will be scanned before you cross the threshold. Travel on Taipei's public transport system, and you will be scanned, and if you have a valid travel card, it will let you onto buses and underground trains without even asking you to take your smart card out of your purse. Technical devices capable of searching you for things you should or should not be carrying are on the increase. We're frisking you for payment cards, stolen goods, weapons, equipment you have no right to remove from your employer's premises - and all without laying a finger on your person. In the airport, the device responsible is a metal 
detector. At the shop or library exit, it is a device for electronic article surveillance, it is a device of a smarter kind presently experiencing rapid sales growth in the surveillance marketplace - a radio-frequency identification (RFID) system. We vary in our operating principles but these devices have one thing in common - pulsed electromagnetic fields (EMFs). We momentarily bathe you in an EMF in order to interact with, and thereby detect, the things We are searching you for - metal objects or magnetic strips or RFID tags. Operating frequencies span a spectrum from tens of hertz to several gigahertzes. And so, like other EMF sources, there is the potential for adverse e $\square$ ects on human health. The Advice Pulsed Fields consortium The aim of the Advice Pulsed Fields project is to survey pulsed-EMF security-system technologies and produce advice for policy makers. We consulted widely with fellow experts, including several involved in the International EMF project coordinated by the World Health Organization (WHO).

The Advice Pulsed Fields research: The first phase of our work was a wide-ranging review of the technical characteristics of pulsed- EMF security systems and consideration of likely future developments. In the second and final phase of the project, we considered the known mechanisms by which electromagnetic fields and biological systems interact and the scientific literature on the e $\square$ ects of EMF exposure on cultured cells, animals, the central nervous system and humans, as well as on electrophysiological stimulation. The team took special care to look into the potential for interactions between EMFs generated by security systems and medical devices. Although not common, damaging interactions of this kind have been reported by patients and confirmed by scientists in laboratory studies. The devices giving most cause for concern are the implanted and portable ones used by ambulatory patients, including implanted defibrillators, heart pacemakers, neurostimulators and drug infusion pumps. Unlike hospital patients, whose environment is controlled, these patients are liable to come into contact with the same variety of pulsed EMFs generated by security systems as anyone else.

The Advice Pulsed Fields results: Since early in 2015, when we adopted our findings its exposure guidelines were not exceeded. On the characteristics of security systems and devices, comprehensive information was not always available. Because of its importance in assessing public and occupational exposures, increased e $\square$ orts at measuring exposure levels were recommended. Another recommendation was that technical information should be provided to purchasers. Information about potential exposure levels should also be routinely taken into account in the design of new systems. Exposure assessment was an area in which the team urged further research, notably to get a better picture of the properties of human tissues in electric fields. A similar preoccupation with information provision pervades the team's recommendations relating to the potential for electromagnetic interference with medical devices. For one thing, more research is required to increase our understanding of the interactions between EMFs and medical devices. The team suggested setting up a European forum in which manufacturers of security systems and medical devices could harmonise our work. For another, We recommended that information about the EMFs generated by security devices be made public to enable informed choices to be made. The ultimate goal, We suggested, should be complete compatibility between security systems and medical devices.

\section{CONCLUSION}

In the irradiation studies, we detected no biological $\mathrm{e} \square$ ects under many exposure conditions. However, in some cases we discovered a change in the ability of certain chemical compounds to flow through artificially created membranes. We also observed genetic damage in lymphocytes (white blood cells) that were directly exposed to $\mathrm{THz}$ radiation without the shielding e $\square$ ect of blood serum. The main conclusion from our work is that $\mathrm{THz}$ radiation will probably not be harmful in biomedical imaging applications, at least when patients are limited to single exposures. However, setting exposure guidelines requires further studies to identify more clearly the relationships between dose and response. The targets for our spectroscopic studies were the various constituents of human blood, such as red and white blood cells, hemoglobin, glucose, total protein and cholesterol, as well as a range of amino acids from which proteins are made. The biological spectra the team collected during the course of the project are now available to research groups worldwide on the project's website1. We also developed tests for analyzing blood samples by infrared spectroscopy of substances such as cholesterol, total protein and glucose. The THz-Bridge survey of THz-radiation users was the first global survey of its kind. Over a period of one 
year, approximately $20 \%$ of the user community returned completed questionnaires. From research groups, these indicate a clear need for action. Not one set of standards for exposure of occupational workers exposed to THz radiation came to light.

\section{Future Scope - Proposed Statistical Model}

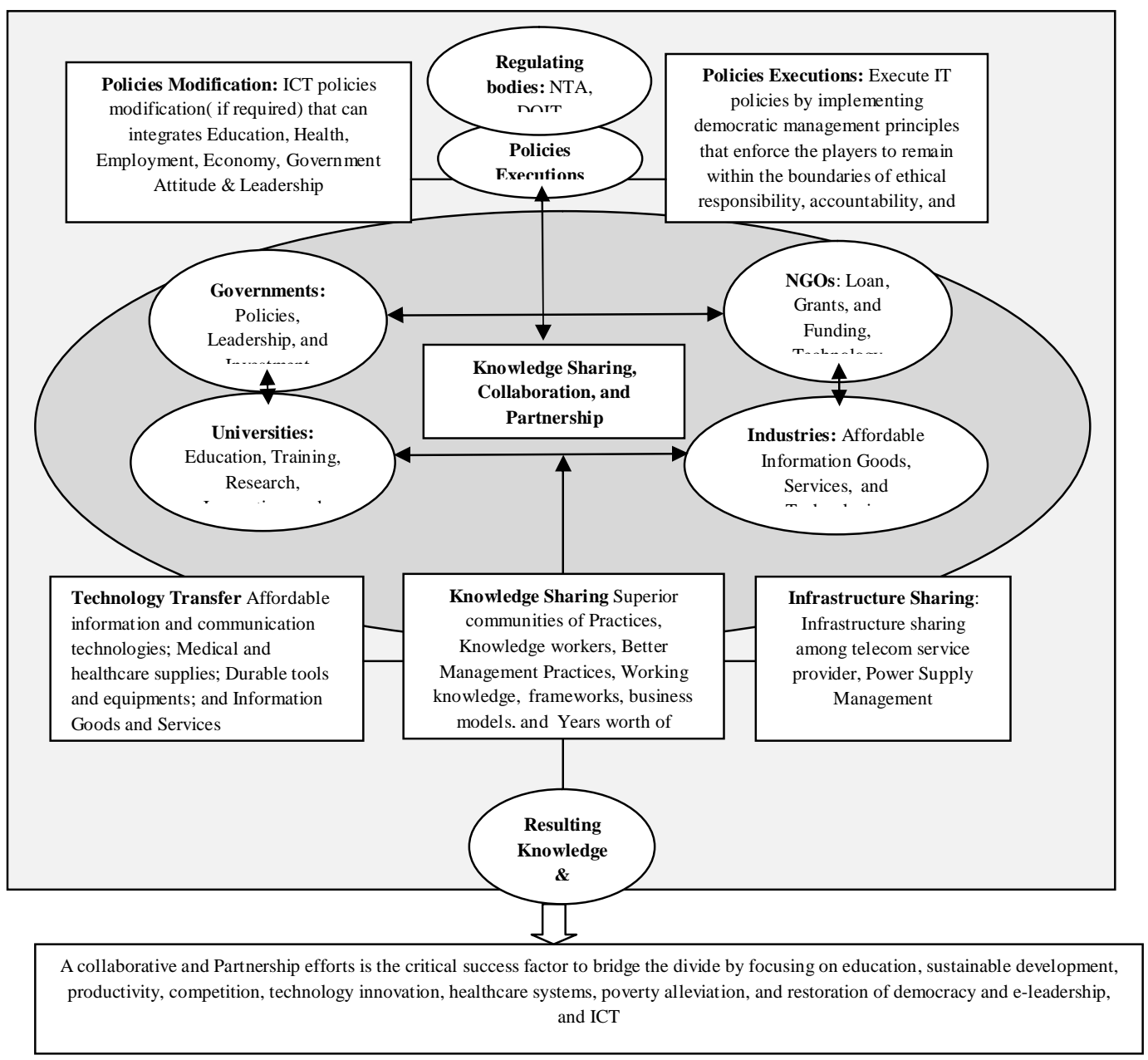

Figure 1 A Strategic Model

\section{REFERENCES}

[1] NTA;" MIS report 2014". (http://nta.gov.np/), 2015.

[2] M. Pun;"Bridging the Digital Divide: the Case of Nepal Wireless". ( UN-APCICT) 2013.

[3] E. Amalie; "How to Bridge the Digital Divide". (Roskilde University Digital Archive) 2011

[4] R. Rice; "Primary Issues in Internet Use: Access, Civic and Community Involvement, and Social Interaction and Expression", (London: In L. Lievrouw \& S. Livingstone) 2002.

[5] L. Chamberlain;"Gender review of ICT projects. Digital Divide Network" (http://www.digitaldividenetwork.org/content/stories/index.cfm) 2002.

[6] Norris, Pippa, "Digital Divide: Civic Engagement, Information Poverty, and the Internet Worldwide, The Press Syndicate of University of Cambridge, USA"2001.

[7] V.H. Carr Jr., " Technology Adoption and Diffusion" (http://www.au.af.mil./au/awc/awcgate/innovation/adoptiondiffusion.htm) 2004.

[8] The Global Information Report 2014: Readiness for the Networked World (GITR), 2014

[9] National Population and Housing Census 2011 (National Report), 2011. 
[10] Measuring the Information Society: International Telecommunication Union(ITU),2013

[11] Fuchs, C., \& Horak, E., "Informational capitalism and the digital divide in Africa. Masaryk University of Law and Technology"; 2013

[12] J Giri "Exploring National and International Approaches to Bridge the Digital Divide and Formulating a Strategic Model That Can Be Implemented in Developing Countries", 2002

[13] https://en.wikipedia.org/wiki/Digital_divide, last access Tuesday, June 23, 2015

[14] http://www.indexmundi.com/nepal/demographics_profile.html last access Tuesday, June 23, 2015

[15] http://cbs.gov.np/ last access Tuesday, June 23, 2015 